\title{
Privacy-preserving Twitter-based Solution for Visually Impaired People
}

\author{
Dina Ahmed Abdraboo \\ Faculty of Computers and Informatics \\ Suez Canal University \\ Ismailia, Egypt
}

\author{
Tarek Gaber \\ Faculty of Computers and Informatics \\ Suez Canal University \\ Ismailia, Egypt
}

\author{
Mohamed El Sayed Wahed \\ Faculty of Computers and Informatics \\ Suez Canal University \\ Ismailia, Egypt
}

\begin{abstract}
Visually impaired people is a big community all over the world. They usually seek help to perform their daily activities such as reading the expired date of food cans or medicine, reading out PIN of a certain ATM Visa, identifying the color of clothes or differentiate between the money notes and other objects with the same shape. A number of IT-based solutions have been proposed to help and assist blind and/or visually impaired people. Generally speaking, these solutions, however, do not support Arabic languages nor protect blind users' privacy. In this paper, Trusted Blind Society (TBS) mobile application is proposed. It is an android application which allows blind users to recognize their unknown surroundings by utilizing two concepts: social networks sites and friendsourcing. These two concepts were employed by allowing family members and the trusted friends, who are registered on Twitter, to answer blind users' questions on a real time. The solution is also bilingual, supports (Arabic/English) and allows screen reader using Android talk-back service. The performance of the TBS system was evaluated using loader.io to check its stability under the heavy load and it was tested by a number of blind volunteers and the results showed good performance comparing to most related work.
\end{abstract}

Keywords-Human powered technology; blind people; visually impaired people; user's privacy; IT-based solution; social networks; friend-sourcing; crowd-sourcing; accessibility; low vision; bilingual; screen reader

\section{INTRODUCTION}

The World Health Organization (WHO) reported that 285 million people around the world are visually impaired (39 million are blind and 246 have low vision) [1]. The huge number of the blind people and the evolution of technology encourage the researchers to introduce solutions to assist blind/visually impaired people to normally practice their daily activities. In the other hand, the social network sites such as Facebook, Twitter, and Instagram, are widely used nowadays and the users of them are growing rapidly. Social networking sites (SNSs) are sites that asset people to communicate together in many ways via the Internet. The target of these websites are not only to interact with each others and making friends but also to gather the information and getting help from known people and share the experiences with them [2]

Arab Social Media Report (ASMR) reported that there are 3.7 million Arab Twitter users [2]. As there are huge number of people in using SNSs and the increase of the Arabic visual impaired, these two important facts motivate us to design and introduce an Arabic application supported by human powered technology while assisting the visually impaired users in identifying the unknown surrounding objects.

Recent solutions are proposed using human-powered technology for assisting the blind such as VizWiz [3]. The VizWiz App allows users to capture an unknown object only one shot to then send to crowd worker who should response with the name of this object. However, using only one shot is not enough because the blind user can't determine the dimensions of the image needed to be recognize. In addition, it is not easy for the blind to capture a clear picture of the unknown object. Other solutions such as Chorus [4], Third Eye [5], they use video to address the limitation of VizWiz but they do not support consumer's privacy as they employ crowd-sourcing concept. In other words, they enable the blind to send the video over Internet and any anonymous helper will get it and help him. Thus, the user shouldn't send any private data to save his privacy.

Human powered technology (friend-sourcing) is the major part of our solution as it considered the identification process while supporting privacy protection to the bind users as well as providing accurate responses to the blind people. Indeed, they can ask anyone for the unknown object but there were some confidential data and situations that he can't share with any strangers except close friend or a family's member [6].

In this paper, Trusted Blind Society (TBS) application is proposed. The TBS is Android-based mobile application enabling blind users to recognize their unknown surroundings by utilizing two concepts: online social networks and friendsourcing. These two concepts were employed by allowing the family members and the trusted friends who are registered on Twitter to answer the blind's private questions on a real-time while other Twitter friends could answer any other questions. The solution also supports two languages (Arabic/English).

This paper is organized as follows. Section II presents the related works while Section III provides the details about the proposed solution. Section IV reports the evaluation of the proposed solution, and Section $\mathrm{V}$ presents the discussion. Finally, Section VI gives the conclusion and future work.

\section{RELATED WORK}

Technology trend aims to found the full automated solutions. However the advances in the technology can't stands alone in facing blind needs such as identification of unknown 
objects supporting their daily activities. For example, OCR failed to read the text on a road sign that captured by mobile phone ${ }^{1}$, object recognition technique worked well with sighted people. However it dosen't work effectively with the blind users. This is because the captured area often isn't clear for the camera [3, 7, 8]. Moreover, screen reader software use some techniques to deliver the content of the screen to the blind, the content is always confusing or unreliable for the blind $[9,10]$.

Instead of automated solutions, disabled people depend on a wide network of friends, volunteers or strangers. An example of that, the volunteer can perform a given task (reading a book for blind) for a certain time of the day but not all the time. Technology evolution engaged the human intelligence and introduces new solutions to assist blind in their daily situations instead of the physically proximate for the helper with the blind. Human-powered access technology [11] has introduced as technology solutions that facilitate the human assistant to help disabled people remotely with flexible support rather than the fully automated solutions that almost not complete or inadequate in daily real situations [12]. Expansion of the human powered solution makes it widely used, therefor researchers deeply study it and classified it into two major concepts: crowd-sourcing and friend-sourcing. The crowd-sourcing enables users to get help from anonymous helpers while friend-sourcing allows the users to get help from known friends.

Indeed, many solutions are proposed to help blind. These include ThirdEye [5], VIZWIZ [3] and LendAnEye [13], and others $[6,13-15]$. These solutions make use of the integration between human resource and the information technology. An overview of these solutions is given below.

\section{- VizWiz}

Bigham et al. [3] introduced an application to address the challenges that faced blinds in their everyday activities (the ability to see food products expired date, recognize ATM ID, etc.). Therefore, they presented an iPhone application called VizWiz that allows blind users to send a picture along with a recorded question about their the unknown object to workers then receive quick answers which are read to the blind users by converting the text to speech. However, VizWiz is not a time efficient solution as sometimes the picture is not clear, thus the helper asks the blind to recapture the image, and repeat the processing again. Moreover, a helper sometimes explains the image content without listening to the question carefully, so the blind would need to repeat the questions again. In addition to the lack of workers available during some time a day. Also, the malicious workers are considered one of the most limitation in this approach [15].

- Third Eye

Lamya et al. [5] discussed the problem for the visually impaired as they faced challenges when practicing their daily tasks. Then, they proposed an mobile application which provides descriptions of all the pictures or videos captured by visually impaired people. The blind can capture an image or record a video

$\begin{array}{ll}{ }^{1} \text { Twitter statistics. 2014,http://www.statisticbrain. } & \text { com/twitter- } \\ \text { statistics/(accessed May } 2015\end{array}$

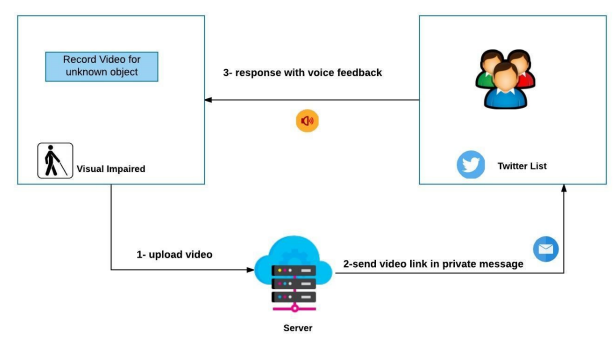

Fig. 1. Data flow of the proposed solution.

about the unknown object then sends it through the server and the volunteer will view and then send his/her feedback as speech to the blind user again. This proposed application presents good performance in recognition as it depends on crowd-sourcing for serving visual impaired requests. However, the privacy factor is not covered as the blind user doesn't know who is going to answer his/her request as it could be stranger volunteers. If the object contains confidential data, such as credit card PIN, it will be known to this strangers.

- LendAnEye

LendAnEye [5] is proposed to introduce a solution for blinds in their daily activates to identify environment surround them. It's a mobile application that has two interfaces one for volunteers and the other one for low-vision user. Unsighted users can contact helpers by double click the screen then a live video call is started, and helper can communicate with the blind via a continuous video call that enhances reality and highly response time. However, the privacy of blind is not guaranteed because helpers are anonymous strangers people the privacy of his request is not achieve. Also this require involving video call service which could be expensive to most of the blind users in the developing countries.

\section{PROPOSED SOLUTION}

Mobile Application called: Trust Blind Society (TBS) is proposed which is an android mobile application that assists the blind in identifying the confidential data. As shown in Fig. ??, the idea of this application is based on the friendsourcing concept while providing help. The user can record a video about the unknown object that he needs to recognize and then sends it over the social network (Twitter) to his selected friend's list. Once the helper identifies the unknown object, he will reply by a message through Twitter, then the TBS will read out the content of the message to the user (using talk back). TBS will access the message of the user Twitter's account through (Twitter API). TBS offers video recording because it's more effective and accurate than the image as the video recording guarantee that the whole range of the unknown object is recorded besides the image may be blurred so the identification process will consume time [3].

TBS is based on friend-sourcing (family members and close friends) which provides the protection of the blind users' privacy as only family members or close friends could see 

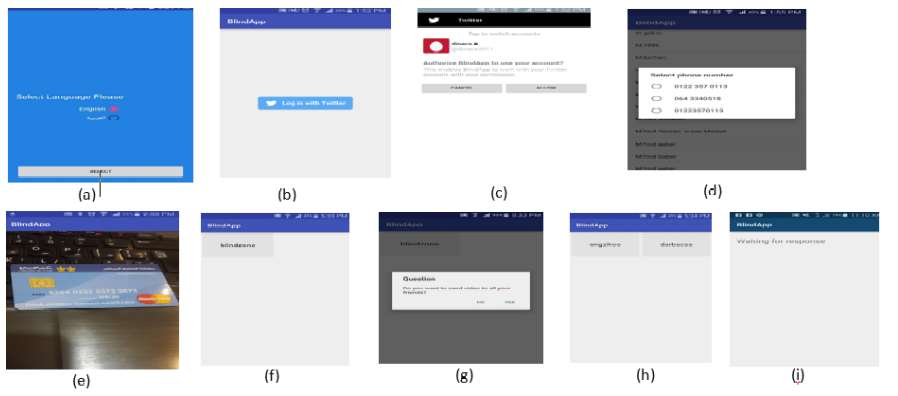

Fig. 2. TBS screen shots.

blind users' private data such as credit card numbers, emails passwords, accounts, private situations that may embarrass him and any private data in his surrounding environment. In other words, TBS integrates the features of (a) Android OS (i.e. Talkback that considered the screen reader), (b) Twitter (i.e. while broadcasting the video to helpers), and (c) friend-sourcing (which is considered the helper side). The implementation of the TBS is done using two main APIs:

- $\quad$ PHP developed APIs: we developed our own APIs for uploading the recorded video to a server.

- Twitter APIs: we embedded twitter API into our application for the social communication while it passes the requested video from the blind user to the helper then get the response back through private message.

These are the main steps, also depicted in Fig. ??, showing how TBS is working:

1 Login to the user Twitter account (through twitter API), to enable the user to access his account data.

2 Select his preferred language (Arabic - English)

3 Select his prefer Twitter's List/ Friends or family members (helper side)

4 Select his featured friend from his phonebook that is embedded in our solution so that when the user has no response up to 30 seconds, a SMS will be sent to his selected friend.

5 Record his video that includes the unknown object and then send it out to the Twitter's list.

6 Read messages for certain conversation as long as the user is $\log$ into his account.

\section{EVALUATION}

The evaluation process for the proposed solution is held to determine if TBS is useful and easy to use or not. The evaluation is done in two ways: target users satisfaction and performance test. Users satisfaction is done to test the functionalities of the solution, the ease of use and accuracy. The performance test is done by using a stress test called, loader.io, to check the response time, evaluate the robustness and availability under the heavy loads.

For the user's satisfaction, 35 blind participants, 13 girls and 22 male, are asked to join our evaluation questionnaire while using our proposed solution. Blinds will access the application trying to identify the unknown object then they will give their feedback about the proposed solution through our questionnaire which consists of the following questions:

- Questionnaire

1 Name, Age

2 Education level

3 Experience with android

4 Is the solution easy to use?

5 Do you think the Application is useful in your daily activities?

6 What do you think is more helpful photo or video?

7 Give some examples in which situations you could use our solution.

8 Rate the solution.

9 Addition ideas to enhance any solution needs more ideas and maintained of it, so this question will assist us in the future work to enhance and present well functional solution.

- Responses

The results of the survey are shown in Fig. ??. Eleven blind users reported that they strongly agree that the application is easy to use and user friendly, while Seven of them agree with this. Only two users are disagree with it because they aren't connected to twitter in addition they suggested to supply them with image recognition technique which would be better for them as they don't want to depend on human factor. In another hand, all of the blind testers agreed that TBS is very secure and save their privacy. So, it could be said that almost $90 \%$ find that TBS is easy to use. Moreover, all of them agree that this application will assist them in their daily activities as they have tested our applications with different examples such as:

- Money identification.

- Password identification.

- Is the makeup matched with the girl?

- Differentiating between colors and paper money.

- If the blind's hit himself he can ask his close friend if it's ok or not).

- Differentiating between boxes that have similar shapes and sizes, like soft drink cans and juice boxes.

- Differentiating between medicine containers and eye drops.

- Checking the expiration dates of different products.

The following is screen shots from videos that recorded via the blind testers (Fig. 3).

In addition, all of the respondents reported that recording a video is useful for them as the video give wide range of the target and they can say all their detailed question while recording (Fig. 5).

- Performance evaluation

Stress test is used to check the performance of our solution under the heavy load of users. We applied it using loaderio software. Stress testing aims to achieve the best performance for websites such as setup static caching servers such as Varnish that enables the software to serve much more users with the same 

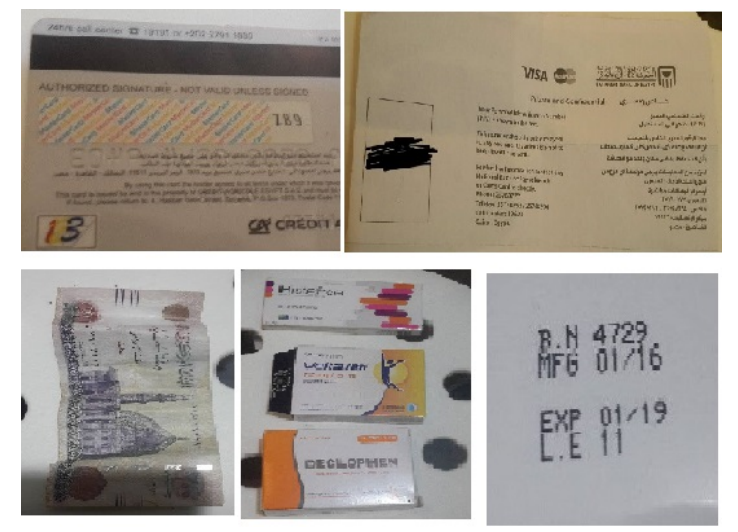

Fig. 3. All screen shots.

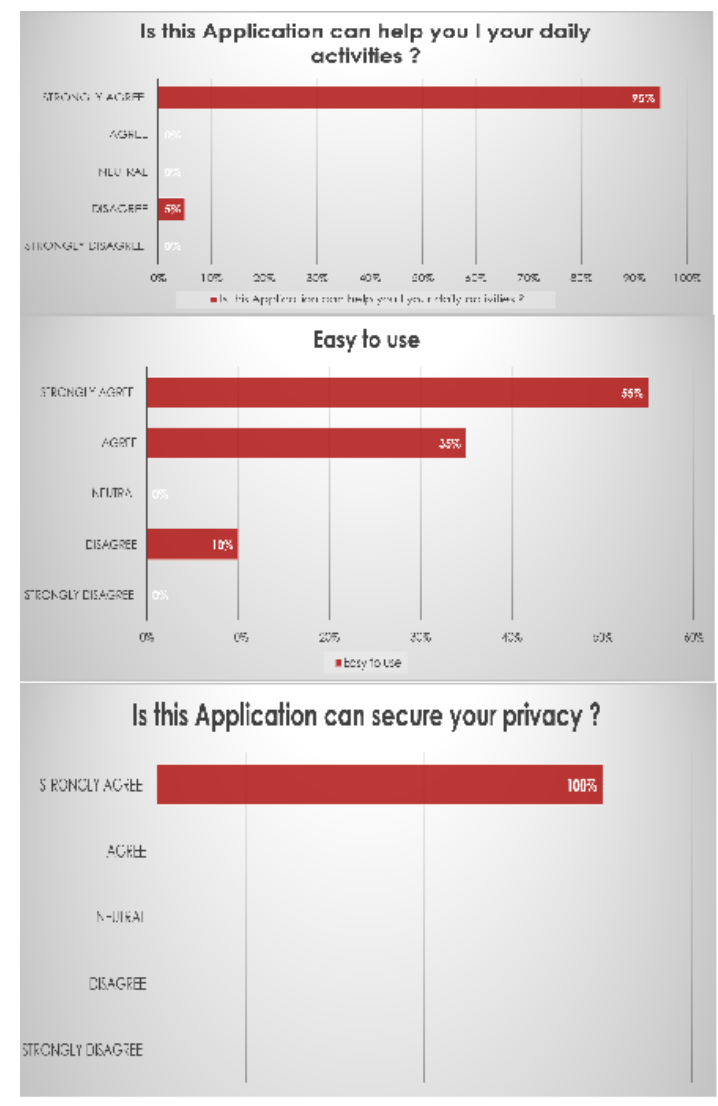

Fig. 4. Evaluation: All evaluation screen shots.

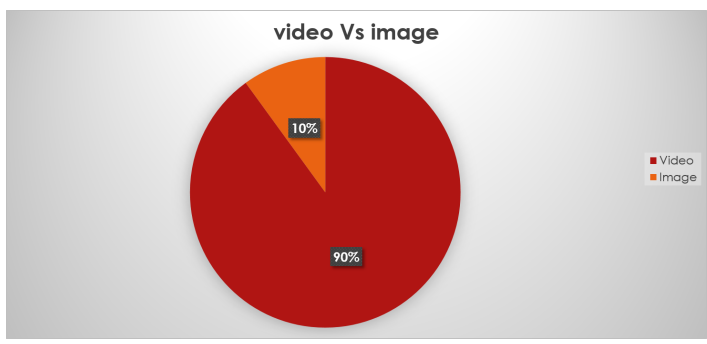

Fig. 5. Evaluation: Video Vs Image.

hardware. Thus the optimization of the website can be held via load testing. Stress testing can be held through

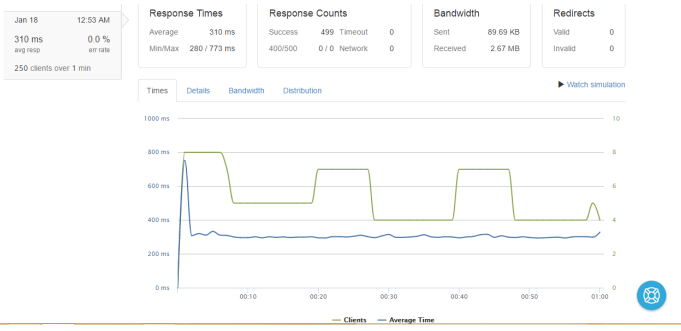

Fig. 6. Stress test simulation about TBS solution by Loader.io.

a new free software tool called Loader.io which is a FREE load testing service that allows you to perform stress test for your web-apps and APIs with thousands of concurrent connections. It simulates connections to our application for certain duration to Monitor and test if the application will perform sufficiently under the load on the application so that the response time can be examined and the availability under the heavy load of the connected people to the application [16]. In Addition, it can measure the response time of our application. We examined if the application can be maintained in a very good way to respond and work well under all circumstances such as (the heavy load of connected users) [17]. The result of applying the stress test to our application is showing in Fig. ??:

\section{Discussion}

The successful results of using the TBS application by blind users means that our TBS app is blind user friendly. This achieved by using auto-focus camera allowing the blind users to feel the vibrating of the mobile when the object is in the camera focus. It was noticed that 30 persons were successfully recorded a video stream about the desired object from the first trial, and 3 of them were successfully completed this cycle from the second trial and two blind users successfully record a video after three trials. it could be said that that the TBS application is easy to use and has friendly interface. Choosing the android as mobile OS was to support the fact that the majority of smart-phones is android-based and they are cheap too. This fact was confirmed by the the volunteers who appreciated the fact that the TBS is an android based smartphone as it is now available with low prices which support the economic case of most blind peoples in Egypt and the Middle East. Moreover, the proposed TBS application is supported with Arabic. So, the TBS application will be much affordable than related applications which are only working at iOS-based smart-phones and only supporting English language.

TABLE I. COMPARISON BetweEn FEATURES OF RELATED SOLUTIONS

\begin{tabular}{|l|l|l|l|l|l|l|l|l|l|}
\hline Feature & VIZWIZ & Chrous-View & ScanSearch & ThirdEye & Legion & SmartEye & EasySnap & LendAnEye & TBS \\
\hline Add Video & $\mathrm{X}$ & $\checkmark$ & $\checkmark$ & $\checkmark$ & $\mathrm{X}$ & $\mathrm{X}$ & $\mathrm{X}$ & $\checkmark$ & $\checkmark$ \\
\hline $\begin{array}{l}\text { Based on friend } \\
\text { sourcing }\end{array}$ & $\mathrm{X}$ & $\mathrm{X}$ & $\mathrm{X}$ & $\mathrm{X}$ & $\mathrm{X}$ & $\mathrm{X}$ & $\mathrm{X}$ & $\mathrm{X}$ & $\checkmark$ \\
\hline Bilingual & $\mathrm{X}$ & $\mathrm{X}$ & $\mathrm{X}$ & $\checkmark$ & $\mathrm{X}$ & $\mathrm{X}$ & $\mathrm{X}$ & $\mathrm{X}$ & $\checkmark$ \\
\hline $\begin{array}{l}\text { Pre-defined known } \\
\text { helpers }\end{array}$ & $\mathrm{X}$ & $\mathrm{X}$ & $\mathrm{X}$ & $\mathrm{X}$ & $\mathrm{X}$ & $\mathrm{X}$ & $\mathrm{X}$ & $\mathrm{X}$ & $\checkmark$ \\
\hline Support Android & $\mathrm{X}$ & $\checkmark$ & $\mathrm{X}$ & $\mathrm{X}$ & $\mathrm{X}$ & $\mathrm{X}$ & $\checkmark$ & $\mathrm{X}$ & $\checkmark$ \\
\hline Free service & $\mathrm{X}$ & $\checkmark$ & $\checkmark$ & $\checkmark$ & $\checkmark$ & $\checkmark$ & $\checkmark$ & $\checkmark$ & $\checkmark$ \\
\hline $\begin{array}{l}\text { Send SMS to pre- } \\
\text { defined number }\end{array}$ & $\mathrm{X}$ & $\mathrm{X}$ & $\mathrm{X}$ & $\mathrm{X}$ & $\mathrm{X}$ & $\mathrm{X}$ & $\mathrm{X}$ & $\mathrm{X}$ & $\checkmark$ \\
\hline \hline
\end{tabular}

A comparison with most related work is conducted and its results are shown in Table ??. From these results, the following 
remarks can be drawn.

- $\quad$ Privacy Privacy factor is not covered in all the previous work. All these work are based on crowdsourcing (anonymous helpers who are not trusted by the blind users). So, they could not be comfortable and feel nonsecured in case of identifying confidential things.

- $\quad$ Supporting offline mode All previous related work are based on the availability of the Internet. However, in the developing countries, the availability of the internet isn't guaranteed. In our solution, this problem has be addressed through sending the video to a close friend's mobile phone using video message.

\section{CONCLUSION}

IT-based solutions supporting the daily life activities of visually impaired people are the most important due to a large number of blind/visually impaired people all over the world. However, most of the current solutions do not support the user's privacy. In this paper, we proposed an assisstive Mobile solution called: Trust Blind Society (TBS), which is an Android application that allows blind users to recognize their unknown surroundings while protecting their privacy. This solution utilized two concepts: online social networks (i.e., Twitter) and friendsourcing. In this solution, the close friend can answer the confidential questions (e.g, bank card security question) while the public friend answers the other questions (e.g., identifying currency notes or food cans). Thus, by utilizing the friendsourcing (friends on Twitter), te privacy of the blind user will be protected. This TBS solution has to be implemented using built-in Android functionalities such as the auto-focus camera for video recording and talk-back accessibility service to read out what is on the screen reader. It also made us of Twitter APIs to handle the connection between the blind users and his/her helpers who have accounts on Twitter. The solution is evaluated in terms of its usability by 35 blind testers. The results was very promising and acknowledged by the testers. Also, the app performance was tested using stress test (loader.io) and the results proved that TBS is stable under the heavy loads and the average response time were $320 \mathrm{~ms}$. In the future, different features can be added to the TBS App and these include: adding automated identification algorithm for basic images, colors and money, supporting $\mathrm{iOS}$ version so that the application will be compatible with the iPhone.

\section{REFERENCES}

[1] M. El-Gayyar, H. F. ElYamany, T. Gaber, and A. E. Hassanien, "Social network framework for deaf and blind people based on cloud computing," in Computer Science and Information Systems (FedCSIS), 2013 Federated Conference on. IEEE, 2013, pp. 1313-1319.
[2] F. Salem, R. Mourtada, and S. Alshaer, "Transforming education in the arab world: Breaking barriers in the age of social learning," Arab Social Media Report, Dubai School of Government-DSG, 2013.

[3] J. P. Bigham, C. Jayant, H. Ji, G. Little, A. Miller, R. C. Miller, R. Miller, A. Tatarowicz, B. White, S. White et al., "Vizwiz: nearly real-time answers to visual questions," in Proceedings of the 23nd annual ACM symposium on User interface software and technology. ACM, 2010, pp. $333-342$.

[4] W. S. Lasecki, P. Thiha, Y. Zhong, E. Brady, and J. P. Bigham, "Answering visual questions with conversational crowd assistants," in Proceedings of the 15th International ACM SIGACCESS Conference on Computers and Accessibility. ACM, 2013, p. 18.

[5] L. Albraheem, R. AlDosari, S. AlKathiri, H. AlMotiry, H. Abahussain, L. AlHammad, and M. Alshehri, "Third eye: An eye for the blind to identify objects using human-powered technology," in Cloud Computing (ICCC), 2015 International Conference on. IEEE, 2015, pp. 1-6.

[6] Y. Zhong, P. J. Garrigues, and J. P. Bigham, "Real time object scanning using a mobile phone and cloud-based visual search engine," in Proceedings of the 15th International ACM SIGACCESS Conference on Computers and Accessibility. ACM, 2013, p. 20.

[7] R. Manduchi, J. Coughlan, and V. Ivanchenko, "Search strategies of visually impaired persons using a camera phone wayfinding system," Computers Helping People with Special Needs, pp. 1135-1140, 2008.

[8] H. Samuel White and J. P. Bigham, "Easysnap: Enabling blind people to take photographs," UIST 2010-Demos, 2010.

[9] Y. Borodin, J. P. Bigham, G. Dausch, and I. Ramakrishnan, "More than meets the eye: a survey of screen-reader browsing strategies," in Proceedings of the 2010 International Cross Disciplinary Conference on Web Accessibility (W4A). ACM, 2010, p. 13.

[10] Y. Borodin, J. P. Bigham, R. Raman, and I. Ramakrishnan, "What's new?: making web page updates accessible," in Proceedings of the 10th international ACM SIGACCESS conference on Computers and accessibility. ACM, 2008, pp. 145-152.

[11] S. Kawanaka, Y. Borodin, J. P. Bigham, D. Lunn, H. Takagi, and C. Asakawa, "Accessibility commons: a metadata infrastructure for web accessibility," in Proceedings of the 10th international ACM SIGACCESS conference on Computers and accessibility. ACM, 2008, pp. 153-160.

[12] J. P. Bigham, R. E. Ladner, and Y. Borodin, "The design of humanpowered access technology," in The proceedings of the 13th international ACM SIGACCESS conference on Computers and accessibility. ACM, 2011, pp. 3-10.

[13] W. S. Lasecki, K. I. Murray, S. White, R. C. Miller, and J. P. Bigham, "Real-time crowd control of existing interfaces," in Proceedings of the 24th annual ACM symposium on User interface software and technology. ACM, 2011, pp. 23-32.

[14] S. White, H. Ji, and J. P. Bigham, "Easysnap: real-time audio feedback for blind photography," in Adjunct proceedings of the 23nd annual ACM symposium on User interface software and technology. ACM, 2010, pp. 409-410.

[15] W. S. Lasecki, R. Wesley, J. Nichols, A. Kulkarni, J. F. Allen, and J. P. Bigham, "Chorus: a crowd-powered conversational assistant," in Proceedings of the 26th annual ACM symposium on User interface software and technology. ACM, 2013, pp. 151-162.

[16] K. Keeker, "Improving web site usability and appeal," Guidelines Complied by MSN Usability Research. Available at http://msdn. microsoft. com/library/default. asp, 1997.

[17] P. Khanna, S. Jain, and B. Babu, "Distributed cloud brokerage: solution to real world service provisioning problems," ARPN Journal of Engineering and Applied Sciences, vol. 10, no. 5, pp. 2011-2016, 2015. 